\title{
Study of antimicrobial effects of honey in comparison to the antibiotics on the microbes isolated from infected burn wounds
}

Quart ul Ain Zahra ${ }^{1}$, Samina Qamer ${ }^{2 *}$, Zahid Mahmood Nagra ${ }^{3}$, Tamsil Ahsan ${ }^{4}$, Raza Hassan $\mathrm{Ch}^{4}$ and Nisar Akber Khan ${ }^{4}$

1. Department of Zoology, Government College University, Faisalabad-Pakistan

2. Honeybee Research Institute, National Agriculture Research Center, Islamabad-Pakistan

3. Department of Plastic Surgery, Allied Hospital, Faisalabad-Pakistan

4. Pathology lab, Allied Hospital, Faisalabad-Pakistan

*Corresponding author's email: saminabee@yahoo.com

Citation

Quart ul Ain Zahra, Samina Qamer, Zahid Mahmood Nagra, Tamsil Ahsan, Raza Hassan Ch and Nisar Akber Khan Study of antimicrobial effects of honey in comparison to the antibiotics on the microbes isolated from infected burn wounds. Pure and Applied Biology. Vol. 5, Issue 4, pp883-888. http://dx.doi.org/10.19045/bspab.2016.50111

\begin{tabular}{llll}
\hline \hline Received: 14/04/2016 & Revised: 03/08/2016 & Accepted: 10/08/2016 & Online First: 22/08/2016 \\
\hline
\end{tabular}

\section{Abstract}

A study was conducted to evaluate the antimicrobial effect of fresh honey produced by Apis mellifera on the bacterial species isolated from infested burns wounds in order to compare it firstly to the antibiotics which are used for the treatment of burn infection. Secondly to assess the possible synergistic effects of bee honey when mixed to antibiotic. Blood specimens were collected with the help of cultural swap from 2-4 weeks old 30 hospitalized patients with burn infection. These blood samples were cultured on different media and four major bacterial strains including E-coli, Klebsiella spp, Staphylococcus aureus and Pseudomonas aeruginosa were isolated and antibiotic sensitivity pattern was measured against these microorganisms by using following three types of antibiotics i.e, Amikacin (AK), Zinacef (CXM), Linozolid (LZD). The result shows that honey has a significant inhibitory growth effects on Pseudomonas aeruginosa's growth (61\%) followed by E. coli (55\%), Staphylococcus aureus (56\%), and Klebsiella spp $(25 \%)$ as compared to the antibiotics alone.

Keywords: Honey; Microbes; Zinacef; Burn wounds

\section{Introduction}

Honey is an antique remedy for infected wounds treatment, which has in recent times been 'rediscovered' by the medical profession, particularly where predictable modern therapeutic managers are failing [1]. Recent evidence also support that honey may actively stimulate healing. In laboratory investigations, honey's antimicrobial action has been found against broad spectrum of bacteria and fungi. Pseudomonas aeruginosa, a notorious organism and showed remarkable resistance to antimicrobial compounds [2]. The use of honey to treat infected wounds is 2000 years old even before the discovery of bacteria as 
a cause of many infections. Honey's inhibitory effect has been reported on about 60 species of bacteria comprising aerobic, anaerobic, gram-positives and gramnegatives [3]. Its antimicrobial activity is due to the osmotic effect, acidity, production of hydrogen peroxide and phytochemical factors in it [4]. Antibacterial activity of honey fluctuates between different types of honey [2]. Honey is a complex product; it has low water activity and acidity, and high sugar content but this does not contribute significantly to the antibacterial activity of those honeys recommended for clinical use. Some of these honeys become more potent upon dilution due to production of hydrogen peroxide generated by the action of glucose oxidase deposited in honey by bees. Addition of catalase to diluted honeys of this type destroys the activity of hydrogen peroxide and reduces or eliminates antibacterial activity. Antibiotics are those molecules that stop microorganisms (both bacteria and fungi) from growing or killing them completely. There are already several non-antibiotic approaches to the treatment and prevention of infection including the use of honey. The sensitivity of organisms to different antibiotics varied depending on the isolate cultured. The present study was designed to have an idea about quick healing of burn wounds either with the use of honey or antibiotics or both.

\section{Materials and methods}

The study was carried out on 30 burn patients, according to the method published [6]. Three Antibiotics viz Linozolid (LZD-

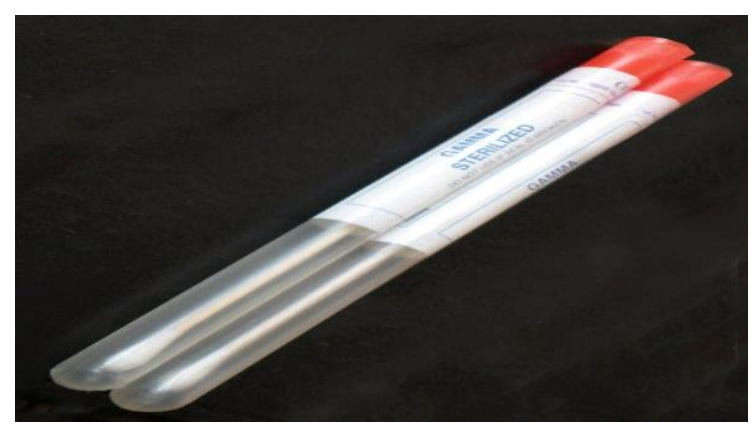

Figure 1a. Cultural swab

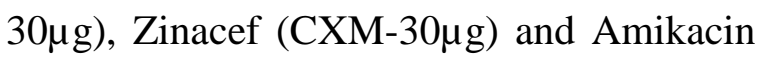
(AK-30 $\mu \mathrm{g}$ ) were selected. The oozing blood samples were taken from the wounds of 2-4 week old burn patients in Burns Unit of Allied Hospital/Punjab Medical College, Faisalabad (Pakistan) with the help of culture sterilized swab (Figure 1a \& b).

Bacterial growth was observed in all samples. Colonies differing in color, shape, size were sub cultured on different agar media such as CLED agar (cysteine lactose electrolyte deficient), blood agar, SS (Salmonella Shiegella) agar, MacConkey agar and incubated again for 24 hours at 37 ${ }^{\circ} \mathrm{C}$. Bacterial colonies were characterized by their growth on different media, hemolysis, lactose fermenting and non- lactose fermenting, morphology and various biochemical tests including Gram's staining, catalase, coagulase, oxidase, vogesproskeur, indole test, methyl red, motility, carbohydrate utilization such as manitol, glucose, lactose. For identification purpose, characteristics of the isolates were compared using a scheme of known taxonomy [7]. Staphylococcus aureus, Pseudomonas aeruginosa, Klebsiella spps and E-coli were identified by biochemical tests. Fresh honey sample of Apis mellifera was taken from beekeeper. A sterile mesh was used to filter and remove residues. As a control, in order to check the microbial purity, the honey sample was cultured on blood and CLED agar plates and then were incubated overnight $\left(37^{\circ} \mathrm{C}\right)$. No bacterial growth was observed (Figure 2).

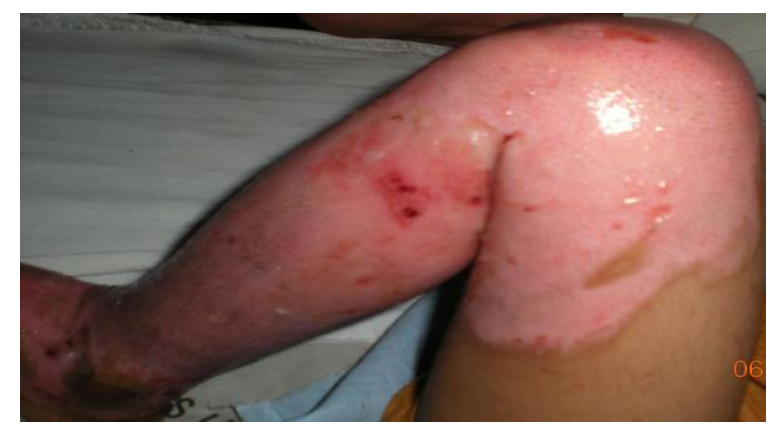

Figure 1b. Burn wound 


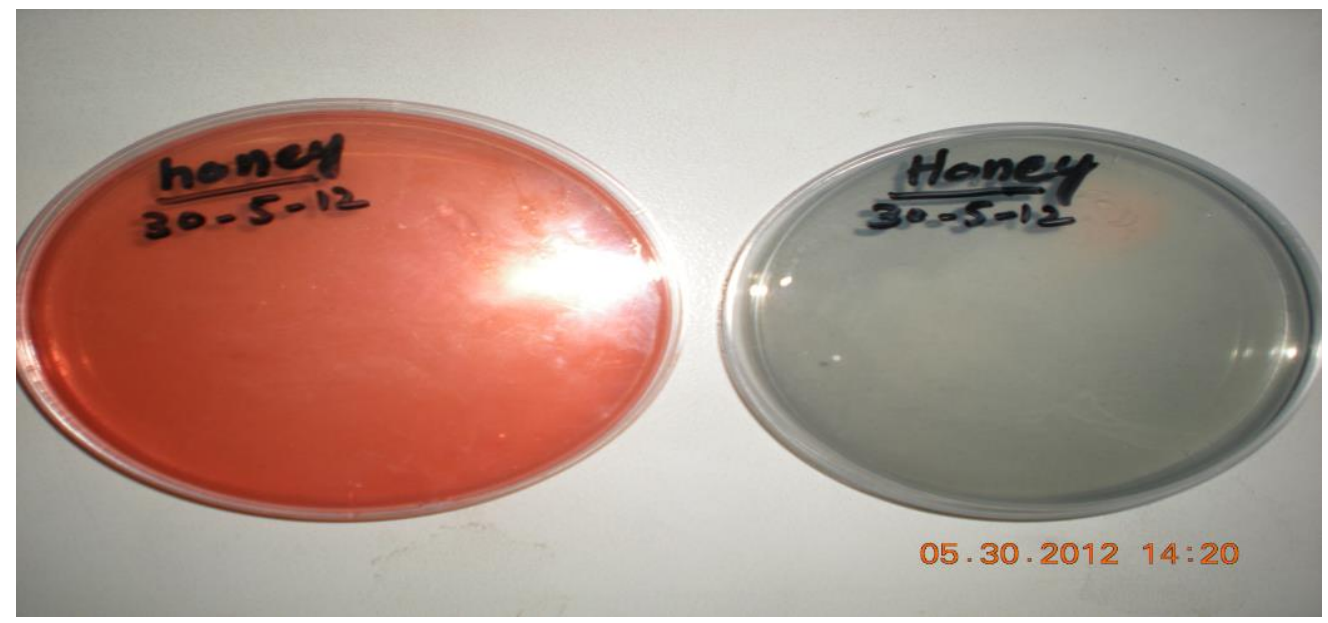

Figure 2. Pure honey cultured on blood and CLED agar (No growth observed)

\section{Sensitivity}

Nutrient broth was prepared and stored in culture plates. Bacterial cultures were pasted on each plate. Divide the plate into 2 halves. On one side of the plate, placed antibiotics discs (Amikacin (AK 30 $\mu$ g), Zinacef (CXM

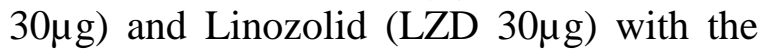
help of sterilized forceps and on the other side (half) of the plate antibiotics along with $50 \mu 1$ honey were placed (just opposite to other half in sequence). On the middle of the plate, a filter paper dipped with honey was placed followed by incubation for 24 hours at $37^{\circ} \mathrm{C}$. Sensitivity of different bacterial strains for honey was measured in such a way that bacterial cultures were pasted on

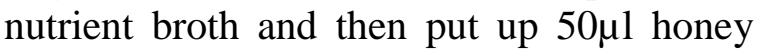
discs (soaked and air dried on a filter paper) with the help of forceps (Figure 3). After that, inhibition zones $(\mathrm{cm})$ were noted on both sides of the plate (antibiotic disc, antibiotic $+50 \mu \mathrm{l}$ honey and $50 \mu \mathrm{l}$ honey (Apis mellifera) alone. The data was subjected to statistical analysis in which different physiochemical parameters were compared by ANOVA [8].

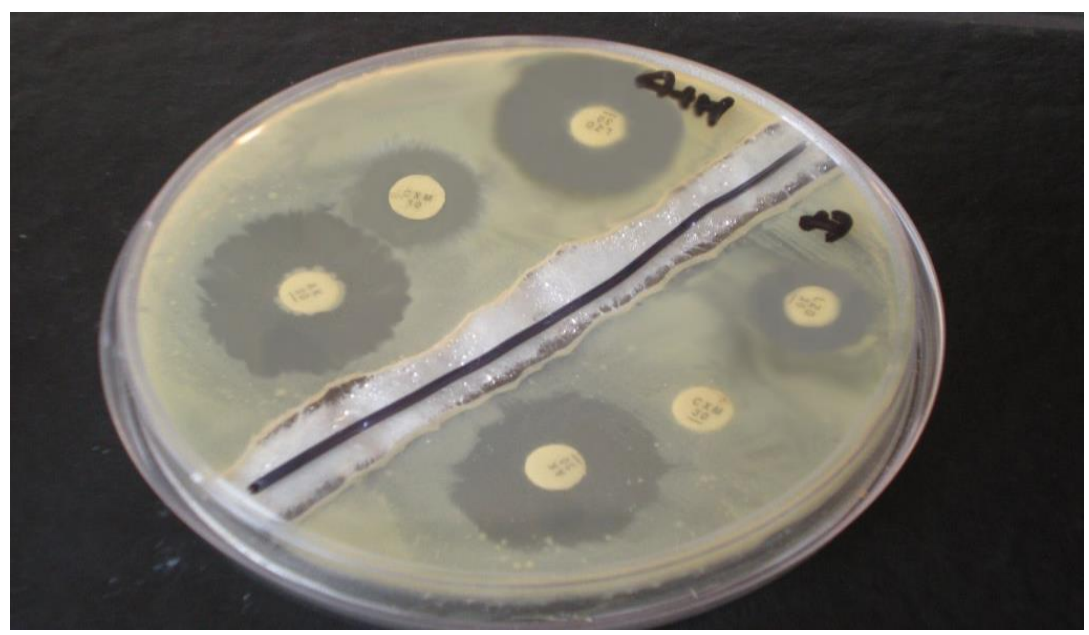

Figure 3. Sensitivity plate. (A) Antibiotics $(A+H)$ Antibiotics + Honey with a filter paper immersed in honey in the centre of the plate 


\section{Results and discussion \\ Bacterial species}

Biochemical tests showed that Klebsiella spp. E.coli and Pseudomonas aeruginosa were gram negative pathogens while Staphylococcus aureus is gram positive. Pseudomonas aeruginosa is reported as the most remarkable bacteria among burn patients which are resistant to antimicrobial complexes [2]. In this study E. coli $(87 \%)$ and Pseudomonas aeruginosa (80\%) were isolated in high percentage followed by Staphylococcus aureus (77\%) and Klebsiella spp (57\%). Same microorganisms are used to investigate the honey antimicrobial activity by others [9].

\section{Sensitivity}

Sensitivity was calculated by measuring zone of inhibitions produced by isolated microbes from burn wounds against antibiotics and honey (Table.1). Klebsiella spp. was isolated on blood agar (Fig: 4) and was found to be more sensitive (14\% for AK, $10 \%$ for CXM and 20\% for LZD) when honey mixed with antibiotics $(50 \mu 1$

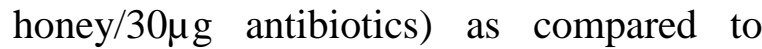
antibiotics $(30 \mu \mathrm{g}$ disc) only ( $11 \%$ for $\mathrm{AK}$, $6 \%$ for CXM and 14\% for LZD) while it was $25 \%$ sensitive for $50 \mu 1$ pure honey (Fig.2). E-coli were isolated on CLED agar. It was $7 \%, 3 \%$ and $8 \%$ sensitive against antibiotics alone while 9\%, 5\% and $13 \%$ with antibiotics (AK, CXM and LZD) and honey mixture. However, pure honey inhibited $55 \%$ of E. coli to propagate. Likewise, Pseudomonas aeruginosa development was prevented $8 \%, 2 \%$ and $8 \%$ by antibiotics (AK, CXM and LZD) only, $10 \%, 3 \%$ and $8 \%$ with mixture of honey and antibiotics and $61 \%$ with pure honey. Similarly, Staphylococcus aureus was isolated on SS agar and antibiotics (AK, CXM and LZD) prevented only 7\%, $4 \%$ and $10 \%$ of its growth, respectively, $0 \%, 6 \%$ and $10 \%$ by the antibiotics when mixed with $50 \mu 1$ honey. Whereas, same amount of pure honey prohibited 56\% of Staphylococcus aureus to grow. CXM proved to be least effective antibiotic compared to $\mathrm{AK}$ and LZD for all four isolated bacterial species under clinical conditions. LZD had maximum synergistic effect of added honey to the antibiotic discs followed by AK and CXM on isolated microbes. A significant increase in growth sensitivity of gramnegative isolates has found when mixed with honey as compared to either antibiotic discs alone or with honey only [6]. According to a study [10] Staphylococcus epidermidis was most sensitive to amikacin. Further a specified noteworthy increased in inhibition zones of antibiotics compounds mixed with honey in comparison to inhibition areas of antibiotics only and same is examined when undiluted pure honey is added to the antibiotics during present study [9]. In another research syne rgistic effect of honey with antibiotics in has been noticed particularly on Pseudomonas aeruginosa, multidrug-resistant microbes and among the commonest bacterium that is the basic cause of infection in burn wounds [12]. The antimicrobial activity of honey on E.coli, Pseudomonas aeruginosa and Staphylococcus aureus is also investigated by [13].

\section{Antimicrobial action of antibiotics and honey on infected burns}

Present study indicates that pure honey could be a significant treatment for infected burns as it inhibits Pseudomonas aeruginosa's growth (61\%) followed by E.coli (55\%), Staphylococcus aureus $(56 \%)$, and Klebsiella spp (25\%). It was observed [14] that honey is capable of eliminating malodors from wounds, eradicating antibiotic-resistant strains of bacteria from wounds and acting as an effective prophylactic agent honey has the potential to be an effective treatment option for burns infected or at risk of infection with $P$. aeruginosa [15]. Commercial 
antibacterial honeys, can inhibit $E$. coli and $P$. aeruginosa [16]. Honey is suggested as an effective natural item for consumption in overcoming the widespread antibiotic resistance of $P$. aeruginosa which is close to the present findings [16].

Table 1. Measurement of inhibition zones $(\mathrm{cm})$ with antibiotics, mixture of honey+ antibiotics and pure honey

\begin{tabular}{|l|l|l|l|l|l|}
\hline \multirow{2}{*}{ S. No } & \multirow{2}{*}{ Antibiotics used } & \multicolumn{3}{|c|}{ Means of inhibition zones (cm) of four bacterial Species } \\
\cline { 3 - 6 } & & $\begin{array}{l}\text { Klebsiella } \\
\text { spp }\end{array}$ & E.coli & $\begin{array}{l}\text { Pseudomonas } \\
\text { aeruginosa }\end{array}$ & $\begin{array}{l}\text { Staphylococcus } \\
\text { aureus }\end{array}$ \\
\hline 1 & AK & $0.49 \pm 0.14$ & $0.51 \pm 0.55$ & $0.59 \pm 0.52$ & $0.52 \pm 0.46$ \\
\hline 2 & AK + Honey & $0.62 \pm 0.3$ & $0.62 \pm 0.3$ & $0.71 \pm 0.48$ & $0.82 \pm 0.52$ \\
\hline 3 & CXM & $0.28 \pm 0.31$ & $0.2 \pm 0.2$ & $0.13 \pm 0.14$ & $0.34 \pm 0.43$ \\
\hline 4 & CXM+ Honey & $0.43 \pm 0.34$ & $0.38 \pm 0.25$ & $0.21 \pm 0.15$ & $0.48 \pm 0.4$ \\
\hline 5 & LZD & $0.63 \pm 0.4$ & $0.57 \pm 0.37$ & $0.54+0.38$ & $0.59 \pm 0.39$ \\
\hline 6 & LZD+ Honey & $0.92 \pm 0.5$ & $0.87 \pm 0.46$ & $0.58 \pm 0.40$ & $0.81 \pm 0.43$ \\
\hline 7 & Honey & $* 1.13 \pm 0.3$ & $* 1.53 \pm 0.44$ & $* 1.4 \pm 0.53$ & $* 1.46 \pm 0.32$ \\
\hline
\end{tabular}

Abbreviations: $* \mathrm{AK}=$ Amikacin, ${ }^{* *} \mathrm{CXM}=$ Zinacef, $* * * \mathrm{LZD}=$ Linozolid, each disc contains $30 \mu \mathrm{g}$ of antibiotic. $\mathrm{P}^{*} \leq 0.05, \mathrm{P}^{* *} \leq 0.05, \mathrm{P}^{* * *} \leq 0.05$

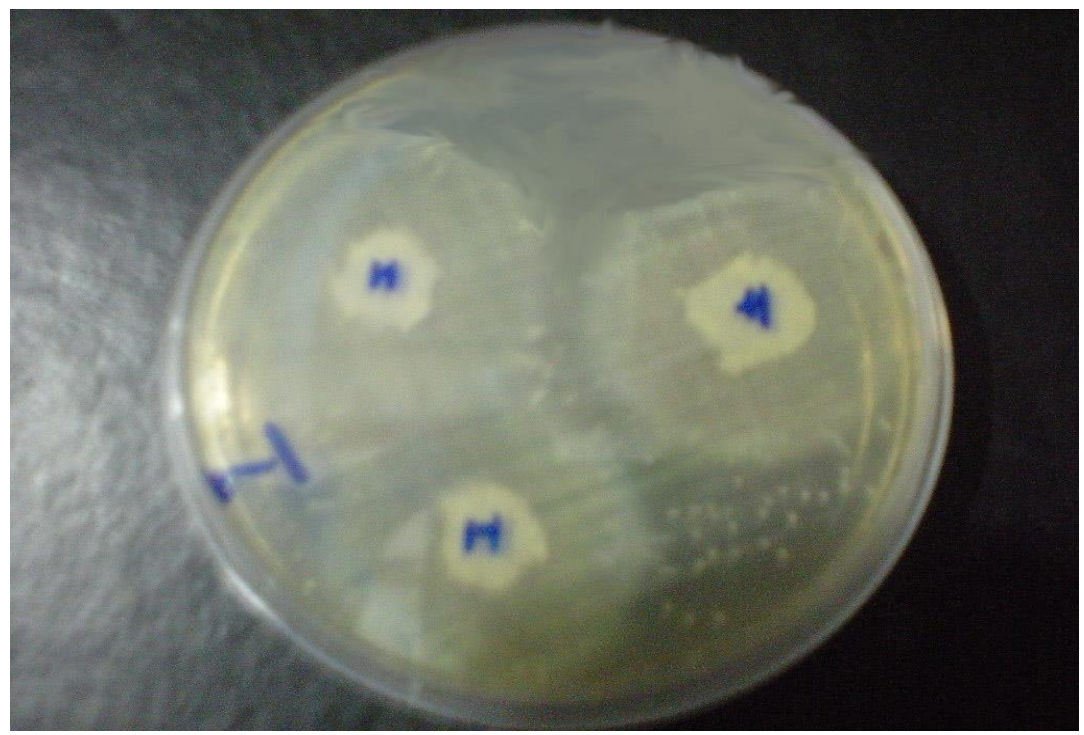

Figure 4. Sensitivity of Klebsiella spp. against honey (H)

\section{Conclusion and recommendations}

The results of this work support that microbial resistance in infected burn wounds is becoming a grave threat and the honey possibly, could represent a reasonable complementary or alternative cure which is cheaply available in all places and is nontoxic. It inhibited the growth of both, gramnegative and positive pathogens. It has an effect which is more bacteriostatic and bactericidal in comparison to certain antibiotics against many wound infecting microorganisms.

Authors' contributions

Conceived and designed the experiments: S Qamer' Performed the experiments: QUA Zahra' Analyzed the data: QUA Zahra\& S Qamer, Contributed reagents/ materials/ analysis tools: ZM Nagra, T Ahsan, RH Ch \& NA khan, Wrote the paper: S Qamer. 


\section{References}

1.Stokes ES, Ridway GI \& Wren GM (1993). Clinical Microbiology, $7^{\text {th }}$ ed. London: Arnold 20-30.

2. Molan PC, Smith IM \& Reid GM (1988). A comparison of antimicrobial activity of some New Zealand honeys. Journal of Apicultural Research 27:252-256.

3.Molan PC (1992). The antibacterial nature of honey; the nature of the antibacterial activity. Bee World 73: 5-28.

4.Jeddar A, Kharsany A, Ramsaroop UG, Bhamjee A, Hafejee IE \& Moosa A (1985). Antibacterial action of honey.

South African Medical Journal 67:257-258.

5.Abd-El A AM, El-Hadidy MR, ElMashad NB \& El-Sebaie AH (2007). Antimicrobial effect of bee honey in comparison to antibiotics on organisms isolated from infected Burns. Annals of Burns and Fire Disasters 20 - n. 2

6.Anwar M, Waqar MA, Khan FA, Tariq Wz, Ahmed S, Mushtaq S, Ahmed Ta, Mirza Sh \& Dawood Mh (2005). Manual of Laboratory Medicine, Armed Forces Institute of Pathology Rawalpindi- Pakistan pp: 127-171.

7.Steel RGD \& Torrie JH (1981). Analysis of Variance factorinal experiments. In: Principles and Procedures of Statistics. $2^{\text {nd }}$ Ed., McGraw Hill International Book Company, Tokyo pp. 336-375.

8.Daniela M (2015). In vitro Study of Honey Antimicrobial Activity. Animal Science and Biotechnologies 48 (1).
9.Dhar S, Sharaf R, Singh K \& Raina B (2007). Microbiological profile of chronic burn wounds among patients admitted in burn unit. Jammu and Kashmir Science 9: 185-87.

10. Cooper RA (2008). Using honey to inhibit wound pathogens. Nurs Times 04(3):46, 48-9.

11. Cooper RA, Halas E \& Molan PC (2002). The efficacy of honey in inhibiting strains of Pseudomonas aeruginosa from infected burns. $J$ Burn Care Rehabil 23(6):366-70.

12. Karayil S, Deshpande SD \& Koppikar GV (1998). Effect of honey on multidrug resistant organisms and its synergistic action with three common antibiotics. Journal of Postgraduate Medicine 44: 93-6.

13. Wilkinson JM \& Cavanagh HM (2005). Antibacterial activity of 13 honeys against Escherichia coli and Pseudomonas aeruginosa. J Med Food 8(1):100-3.

14. Adeleke OE \& Olaitan PB (2006). The anti-pseudomonal property of honey and gentamicin. Annals of Burns and Fire Disasters XIX -3.

15. Al-Waili NS (2004). Investigating the antimicrobial activity of natural honey and its effects on the pathogenic bacterial infections of surgical wounds and conjunctiva. Journal of Medicinal Food 7: 210222.

16. Kamel AH \& El Megeed EA (1997). The role of aztreonam in the control of gram negative burn wound infection. Annals of Burns and Fire Disasters 9: n. 1. 